\title{
Measuring of Surface Shape of Cloths by Ultrasonic Sensor
} By Shigeru Nishikawa, Haruo Niwaya, Atsuo Shibuya and Noboru Aisaka

\author{
Research Institute for Polymers and Textiles, Tsukuba, Ibaraki \\ Based on the Journal of the Textile Machinery Society of Japan, Proceedings, Vol. 40, No. 4, P174-P180(1987-4)
}

\begin{abstract}
We have tried applying the distance mesuring technique by ultrasonic sensor to non-contact measurement of the surface shape of cloth with small curve such as wrinkle. An ultrasonic sensor used is a focusing type (dia.: $30 \mathrm{~mm}$; focus: $50 \mathrm{~mm}$; beam dia.: $1 \mathrm{~mm}$ ). It is observed that the sensor can detect the change at the level of $1 \mathrm{~mm}$ both in height and in interval of the curved surface and this measuring method has the possibility to obtain the fairly accurate result for the measurement of the shape of small curved surface.

Also, we have tried measuring the 3-dimensional shape of garment made of some large curved surfaces. In the experiment, we measured the distance from reflecting surface by attaching a sensor (dia.: $10 \mathrm{~mm}$; focus: $60 \mathrm{~mm}$; beam dia.: $6 \mathrm{~mm}$ ) at the end of the arm of drape measuring apparatus (trial model). In this measurement, the data of 3-dimensional shape of garments made of soft and thin materials were easily detected, and the efficiency of a non-contact measuring method by the ultrasonic was proved.
\end{abstract}

\section{Introduction}

Non-contact measurement of 3-dimensional shape is advantageous in cases such as wrinkles appeared on the cloth or the curved surface formed by sewing. At present, the practical non-contact measuring methods are the Moire' Camera system ${ }^{[1]}$ of projection type and laser measuring system ${ }^{[2]}$ developed for the measurement of body size and the 3-dimensional shapes made of a large curved surface like garments. On the other hand, several optical measuring methods ${ }^{[3]-[7]}$ are introduced to the comparatively small curved surface such as wrinkle and seam puckering.

The aim of conventional optical methods is applied to check the height and the number of the wrinkles for the purpose of quantitative evaluating. Therefore, this method is imperfect when shape of individual wrinkles are measured. Moreover, there is an essential problem in the optical measuring methods; the influence by the color shade of the surface of cloth.

This research is an attempt of an application of the ultrasonic technique on the basis of the distance measuring method, to measure the surface shape of cloth with small curved surfaces. In the experiment, we discussed the measurement of the small uneven shape on the cloth surface by applying an ultrasonic sensor of beam focusing type. Furthermore, as an application measurement, we tried measuring the 3-dimensional shape of garments.

\section{Method of the Distance Measuring by Ultrasonic Waves}

The principle of the measurement is that the distance to an object is calculated by the required time of reflecting the ultrasonic waves from the surface. Namely, distance $L$ (cm: one way) to the object surface will be given in formula (1)

$2 L=V T$

where $T(\mathrm{sec})$ is the reflecting time of the ultrasonic waves, and $V(\mathrm{~cm} / \mathrm{sec})$ is the transmitting speed of the ultrasonic waves in the air. When the temperature of air is given with $t^{\circ} \mathrm{C}$, distance $L$ is shown by

$L=T(61 t+33,100) / 2$

Where $T$ is the time when intensity of the received wave arrives at a certain level (threshold intensity). $T$ is measured by applying clock pulse. When the number of pulse is $N$ and the frequency of pulse is $f^{\prime}, T$ is given in $T=M / f$. Thus, when temperature $t$ is $20^{\circ} \mathrm{C}$ and frequency $f$ ' is set up to $3.432 \mathrm{MHz}$, formula (2) is given as follows.

$L=N / 200$

Accordingly, the distance $L$ to an object surface is instantly obtained by counting the number of clock pulse. However, the error of the ultrasonic speed by the 
temperature change needs to be compensated always in the measurement.

\section{Outline of Measuring Apparatus of the Surface Shape of Cloths (Trial Model)}

Figure 1 shows the appearance of the apparatus which was tried for this research. It is composed of an ultrasonic sensor unit, a sensor scanning unit, a signal processing unit and an operating box. The ultrasonic sensor unit has a sensor of concave piezoelectric transducer type having both functions of reception and transmission of the ultrasonic waves.

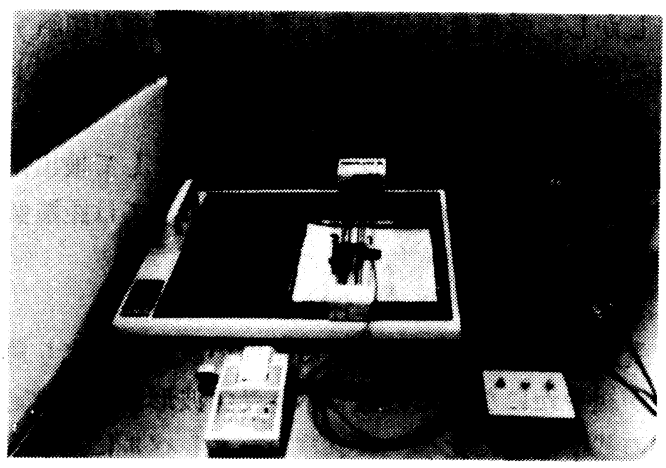

Fig. I Measuring apparatus of surface shape of cloth (trial model)

In this measurement, we applied two types of sensors namely A type and B type. As shown in Table l, A type is $10 \mathrm{~mm}, 6 \mathrm{~mm}$ and $B$ type is $30 \mathrm{~mm}, 1 \mathrm{~mm}$ in outside diameter, beam diameter respectively. Figure 2 shows
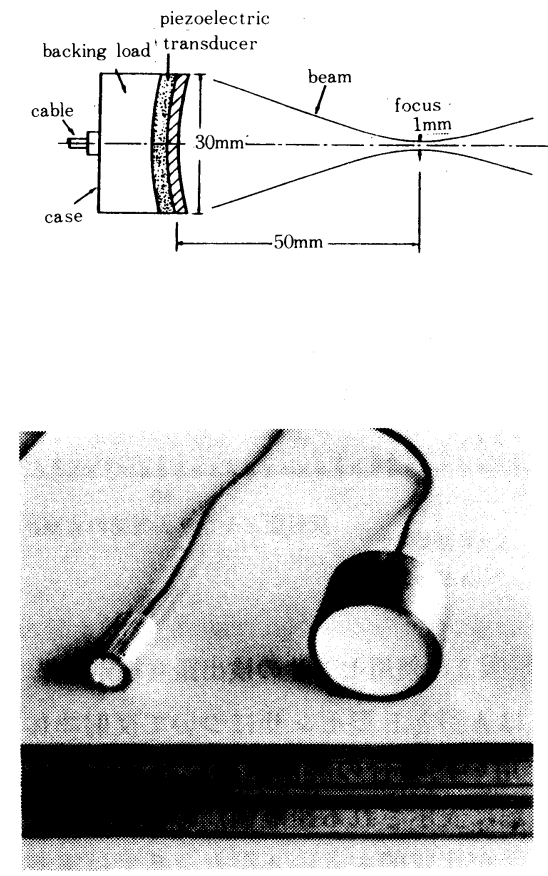

Fig. 2 Appearance and structure of sensor
Table 1 Dimensions of sensor

\begin{tabular}{l|c|c|c|c}
\hline Type & $\begin{array}{c}\text { Dia. } \\
\mathbf{D} \\
(\mathrm{mm})\end{array}$ & $\begin{array}{c}\text { Focal length } \\
\mathbf{F} \\
(\mathrm{mm})\end{array}$ & $\begin{array}{c}\text { Beam dia. } \\
\mathbf{d} \\
(\mathrm{mm})\end{array}$ & $\begin{array}{c}\text { Frequency } \\
\mathbf{f} \\
(\mathrm{kHz})\end{array}$ \\
\hline \hline A & 10 & 60 & 6 & 910 \\
\hline B & 30 & 50 & 1 & 882 \\
\hline
\end{tabular}

those appearance and a structural drawing of the B type sensor. The sensor scanning unit has the mechanism which moves the sensor to $X, Y$ directions. The signal processing unit carries out the modulation of the received and transmitted waves, the count of clock pulse, the conversion of the reflecting time into the distance, and the digital display of the distance. The operating box has manual operating switches which command the movement to $X, Y$ directions of the sensor.

In this experiment, we applied a miniature lathe as the sensor scanning unit. It was used to improve the accuracy of measuring position of the sample.

In the experiment, it was necessary to determine an standard distance preliminarily. Therefore, a glass plate was used as a reflector and this put at the position of $\mathbf{5 0 . 0}$ $\mathrm{mm}$ from the sensor surface. Then, the threshold level was adjusted so that detecting distance is displayed as $50.0 \mathrm{~mm}$. In this case, the levels in the $\mathrm{A}$ and $\mathrm{B}$ type sensors are $0.19 \mathrm{~V}$ and $0.20 \mathrm{~V}$ respectively.

\section{Experiments and Discussion}

\subsection{Range of the detecting distance}

The sound intensity which is transmitted in the air from a piezoelectric transducer attenuates due to scattering by the reflector, absorption by the air, and the diffusion of the ultrasonic beam in the transmission. The sound pressure of received wave (maximum amplitude) reduces by such phenomena. Accordingly, the distance range where the measurement is effective is inevitably limited.

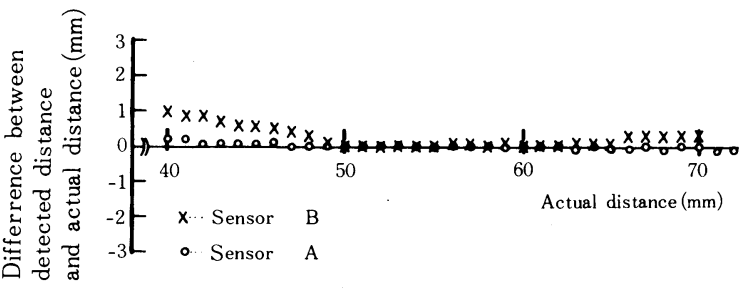

Fig. 3 A test result of detected distance by sensor

Figure 3 shows the test result of the distance detected by the glass plate used as a reflector for the A and B type sensor. The result of A type sensor agree to an acutual distance in a wide range $(30 \mathrm{~mm})$. However, the effective range of $B$ type sensor is only $16 \mathrm{~mm}$. In the sensor of small beam diameter, it is shown that the measurement is limited in a very small range. 
4.2 Detecting ability of sensor for small unevenness

Judging from the focus beam diameter of B type sensor, it is estimated that the size of detecting limit of the sensor is about $1 \mathrm{~mm}$. In order to prove this limit, we measured a board on which straight steel wires of $0.8 \mathrm{~mm} \phi$ are arranged at the interval of $0.8 \mathrm{~mm} \phi$. Figure 4 shows the distance which were able to detect straight steel wires at a measuring distance of $51.5 \mathrm{~mm}$ to the board surface. As shown in Fig. 4, it proves that amplitude and wave length of the curve almost coincides with the diameter of wire and the interval of the arrangement. Accordingly, it is recognized that the detecting ability of the $B$ type sensor is longer than $0.8 \mathrm{~mm}$.

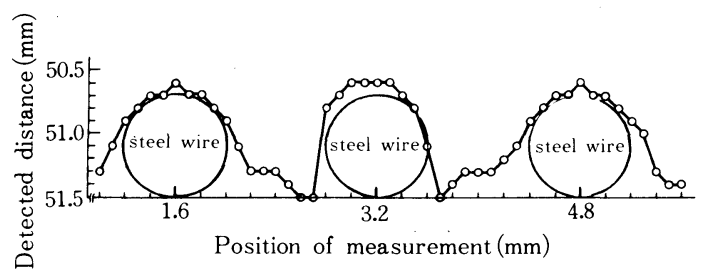

Fig. 4 Detected results for the wires

\subsection{Error of detecting value caused by decrease of reception level}

When the amplitude of received waves decreases, the rising shape of the envelope of waves shows more lenient inclination. By this reason, there is a time delay $(\Delta T)$ until received wave reaches to the threshold level. Therefore, an error $(\Delta L)$ corresponding to $(\Delta T)$ is added into the detected distance $L$.

Figure 5 shows the reflecting time error $(\Delta T)$ appeared between the standard reflected wave and the reflected wave which caused under $11.0^{\circ}$ inclination of the reflector at the distance of $50.0 \mathrm{~mm}$. In this case, the detecting distance is indicated as $51.1 \mathrm{~mm}$.

The detecting distance errors caused by the attenuation of reception level are shown in Fig. 6. From the results, it is evidence that the error included in the detecting value increases together with the decrease of reception level.

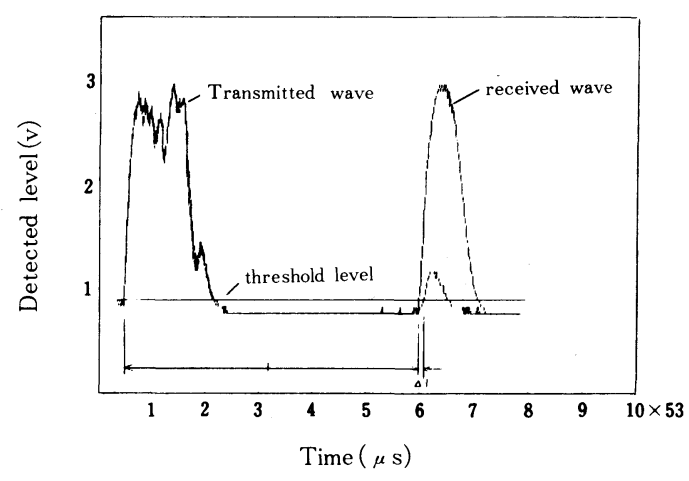

Fig. 5 Reflected time error produced by lowering of level of received waves

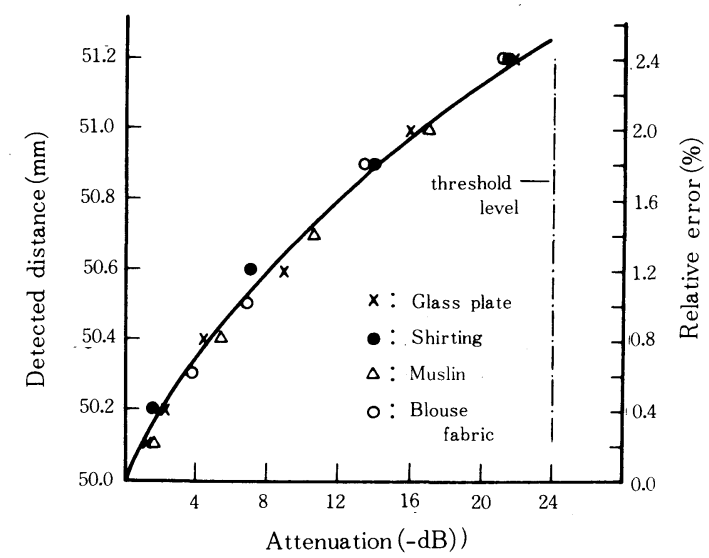

Fig. 6 Relation between distance error and attenuation of received waves

\subsection{Ultrasonic reflection and transmission charac- teristics of cloth}

We examined the ratio (attenuation) of the reception intensity by cloth to the standard reception intensity in order to compare the ultrasonic reflection and transmission characteristics of cloth.

In the experiment, the sample is put between A type and $B$ type sensors, in order to detect the reflected and transmitted waves at the same time.

A type sensor has both functions of emission of ultrasonic waves and reception of reflected waves. B type sensor receives only emitted wave from the A type sensor. The A type sensor is put at the position of $50.0 \mathrm{~mm}$ from the testing sample and the B type sensor is put at the position of $5.0 \mathrm{~mm}$ from the testing sample on the opposite side. The standard intensity of reflected waves is the maximum amplitude of the waves received by A type sensor when a glass plate is put at the same position from the testing sample. And, the standard intensity of transmitted waves is equal to the maximum amplitude of the waves when the B type sensor receives the waves from the A type sensor directly without the testing sample.

Table 2 shows the attenuation of ultrasonic reflection and transmission of the several testing samples. It is clear that as shown in Table 2 for the testing samples (a) and (b), the attenuation of ultrasonic transmission are large because these textile structure are thick and compact.

The reflection attenuation of testing sample (c) is large because of the reflection loss caused by hair feather on the cloth surface. Also, the transmission attenuation of testing sample (d) is extremely small by the reason of thinness of the cloth.

\subsection{Measurement of wrinkle shape of cloth}

In this experiment, we used cotton a shirting as the testing sample. The wrinkles were given to the cloth by natural drying after wet processing. The size of testing sample is about $70 \times 70$ in $\mathrm{mm}$, and its edges were fixed to a frame. As a preliminary examination, we investigated the ultasonic reflection and transmission characteristics of the testing sample from which the wrinkles were removed by ironing. The B type sensor was used in the test. As shown in Table 2 , transmitted waves were hardly detected 
Table 2 Specification and ultrasonic characteristics of the testing samples

\begin{tabular}{|c|c|c|c|c|c|c|c|c|c|}
\hline \multirow[t]{2}{*}{ Symbol } & \multirow[t]{2}{*}{ Sample } & \multirow[t]{2}{*}{ Material } & \multirow[t]{2}{*}{ Design } & \multirow{2}{*}{$\begin{array}{l}\text { Weight } \\
\left(\mathrm{g} / \mathrm{cm}^{2}\right. \\
\left.\times 10^{-2}\right)\end{array}$} & \multirow{2}{*}{$\begin{array}{c}\text { Thickness } \\
(\mathrm{mm})\end{array}$} & \multicolumn{2}{|c|}{ Density (yarn/cm) } & \multicolumn{2}{|c|}{ Attenuation $(-\mathrm{dB})$} \\
\hline & & & & & & Warp & Weft & Reflection & $\begin{array}{l}\text { Transmis- } \\
\text { sion }\end{array}$ \\
\hline a & $\begin{array}{l}\text { Worsted } \\
\text { fabric }\end{array}$ & Wool & $3 / 3$ twill & 2.78 & 0.71 & 21 & 17 & 11.8 & 12.6 \\
\hline b & $\begin{array}{l}\text { Shirting } \\
\text { (raw) }\end{array}$ & Cotton & Plain & 1.54 & 0.66 & 23 & 23 & 2.7 & 6.0 \\
\hline c & $\begin{array}{l}\text { Coat } \\
\text { fabric }\end{array}$ & Wool & Plain & 2.81 & 1.68 & 8 & 7 & 12.3 & 4.7 \\
\hline d & Georgette & PET & Plain & 0.80 & 0.23 & 42 & 37 & 9.8 & 0.4 \\
\hline e & Muslin & Wool & Plain & 1.04 & 0.32 & 30 & 26 & 6.0 & 2.5 \\
\hline $\mathrm{f}$ & $\begin{array}{l}\text { Blouse } \\
\text { fabric }\end{array}$ & PET & Plain & 0.83 & 0.24 & 66 & 45 & 6.3 & 1.6 \\
\hline $\mathrm{g}$ & $\begin{array}{l}\text { Shirting } \\
\text { (wet/dry) }\end{array}$ & Cotton & Plain & 1.92 & 0.72 & 27 & 25 & 1.2 & - \\
\hline
\end{tabular}
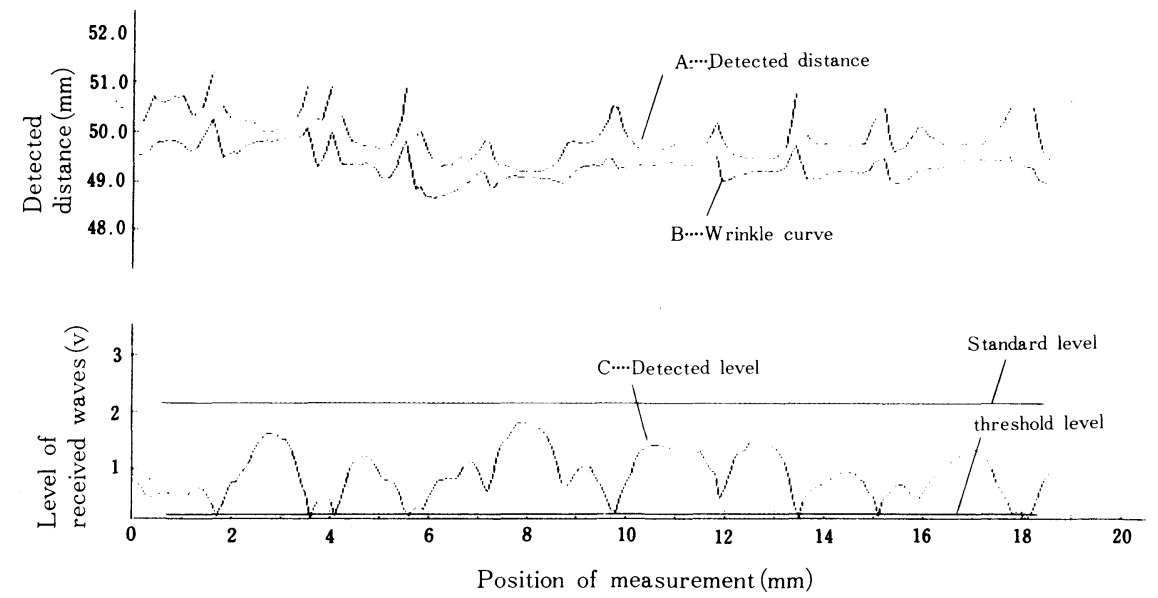

Fig. 7 Measuring result of the surface shape of the shirting with wrinkles

because the structure of the textile became more compact by such a wet type processing (testing sample: g). Also, the detecting distance was not affected by the unevenness of cloth surface by crossing of warp and weft.

Figure $7(\mathrm{~A})$ is the distance detected with $0.1 \mathrm{~mm}$ notch along weft direction of the cotton shirting with wrinkles. Figure $7(\mathrm{C})$ shows the maximum amplitude of received waves. Under the phenomenon an shown in Fig. 7(C), it is clear that the increase of the error is shown conspicuously, because such a fluctuation of the amplitudes depends remarkably on the angle of inclination of the measured surface. Moreover, the distance is not detected in the position where inclination angle is extremely large. Accordingly, it was hard to think that Fig. 7(A) shows the exact wrinkle shapes.

Figure $7(\mathrm{~B})$ shows the wrinkle shapes of the cotton shirting. This is a curve obtained by compensating for the relation between amplitude of received wave and distance errors (Fig. 6), and interpolating at the impossible points of detection on the basis of Fig. 7(A).

\section{Application to 3-dimensional Shape Measurement of Garments}

For the measurement, we used a drape meauring apparatus $^{[8]}$ which was experimentally produced in our laboratory. The ultrasonic sensor is attached at the end of the measuring arm of this apparatus. We tried non-contact measuring the 3 -dimensional shape garment by this device. Fig. 8 shows the ultrasonic sensor attached at the end of the measuring arm.

\subsection{Constitution of measuring device and measuring method}

The device consists of the rotational driving unit where a dummy is attached to its axis, the measuring arm which has 3 sets of sticks and joints, and the rotational driving control unit which decides measuring angle of the testing sample. Also, the main measuring part of this device consists of the measuring unit which detects ultrasonic 
signals by the sensor, the signal processing unit which processes the ultrasonic signals, and the data processing unit which computes the measured positions and shows the shapes on the graphic display.

A measuring arm is slided manually so that a beam focusing range of the ultrasonic sensor reaches on the surface of the testing sample. And, the distance detected by the sensor, the movement of the measuring arm, the turning angle of each joint, and the rotational angle of the testing sample, are detected as the data. These data are transmitted to the data processing unit, where the coordinates of the measured position are caluculated. The measurement is carried out at an interval of certain rotational angle around the testing sample, and then the cross section is shown on the graphic display. Subsequently, this operation is proceeded at an interval of certain height of the testing sample, and the final result of the measurement is shown as the 3-dimensional shape on the graphic display.

Of the three joints, two are attached horizontally and surement is shown as the 3-dimensional shape on the inclined incidence of transmitted waves, by making free with the direction of the sensor surface.

\subsection{Results and discussion}

When measuring the large curved surface of the testing sample, it was advantageous to use the sensor of large focusing range and wide beam diameter. Accordingly, the A type sensor was used for this measurement.

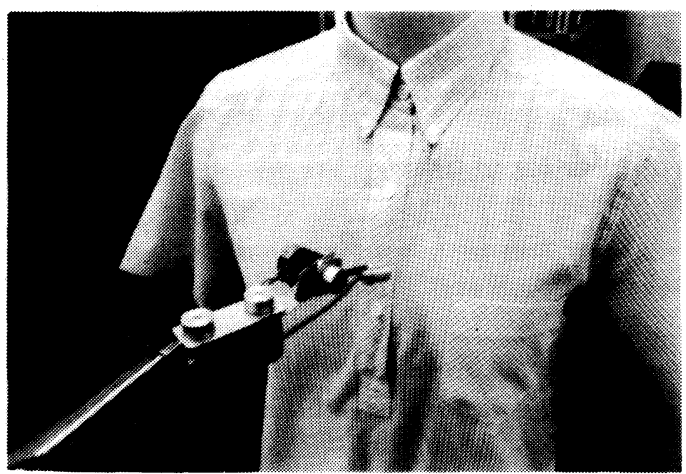

Fig. 8 Measuring arm installed ultrasonic sensor

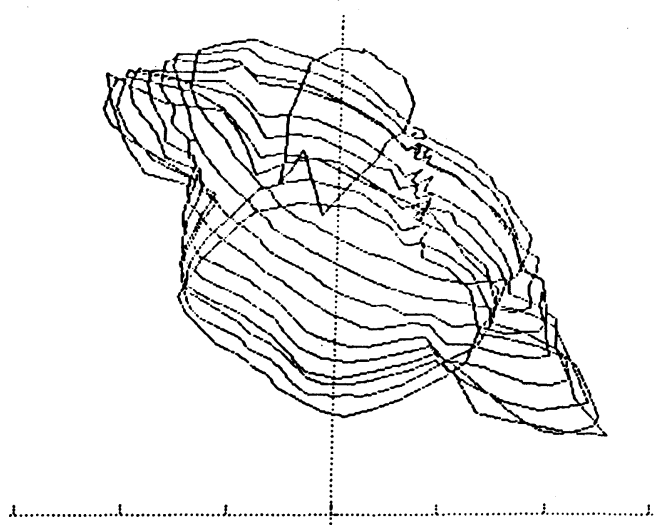

As an example, the measuring result of cotton blouse (a testing sample of Fig. 8) is shown in Fig. 9. This is a 3dimensional shape which is measured at $30 \mathrm{~mm}$ interval in height and $5^{\circ}$ interval in rotational angle.

In case of a sample with flat surface and large curvature radius, the reflected waves were strong even if the joints were not operated, and measuring efficiency was good. In the testing sample having mixed with concave and convex surfaces, the data can only be obtained by operating the three joints. In the measurement of garment of such a shape, the efficiency of present device is not satisfactory, because the arm and joints were operated by hands. Usually, the measuring time required for one cross section is about 8 minutes under $5^{\circ}$ interval in rotating angle, but it takes about 15 minutes in case that the joint operation is required.

Thus the non-contact measuring method by ultrasonic sensor was effective for the garments with complicated shapes.

\section{Conclusion}

Regarding curved surface of garments and wrinkle shape of cloth, we tried non-contact measuring method by the ultrasonic waves. In our experiments, it was shown that the small unevenness of the shapes can be measured by the sensor of beam focusing type and we could obtain the sufficient advantage of non-contact measuring method in the measurement of 3-dimensional shape of garments.

Originally, the sensing technique using the ultrasonic waves has many advantages such as the easiness of operation, non influence of environment noise, directivity of the ultrasonic waves, and so on. When being applied to the cloth as an object of the measurement, this method has a large merit that color of cloth does not affect on its detection. The utilization of sensing technique by the ultrasonic waves is expected as a powerful method of measurement for the rationalization of the textile process.

\section{References}

[1] S. Nishikawa, N. Suda; Bull. Res. Inst. Polym. Text., No. 142, (1984).

[2] Technical Report, Japan Small Business Cooperation (1985).

[3] S. Suzuki, K. Suzuki; Instrumentation and Automation, 5, 5 (1977).

[4] N. Kusakabe et al.; J. Japan Res. Assn. Text. End-Uses, 14, 9 (1973).

[5] R. B. Belser et al.; Text. Res. J., 38, 315 (1968).

[6] R. S. Hunter, C. A. Lofland; Am. Dyest. Rep., 48, 54 (1969).

[7] J. R. Bercaw; Am. Dyest. Rep., 49, 111 (1960).

[8] S. Nishikawa et al.; Prep. Ann. Conf. Res. Inst. Polym. Text., (1984).

Fig. 9 Three-dimensional shape of garment measured by ultrasonic sensor 